\title{
Synchronization between arterial blood pressure and cerebral oxyhaemoglobin concentration investigated by wavelet cross-correlation
}

\author{
A. B. Rowley ${ }^{1,3}$, S. J. Payne ${ }^{1}$, I. Tachtsidis ${ }^{2}$, M. J. Ebden ${ }^{1}$, J. P

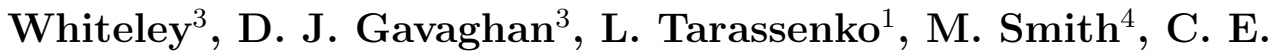 \\ Elwell $^{2}$, and D. T. Delpy ${ }^{2}$ \\ ${ }^{1}$ Department of Engineering Science, University of Oxford, Parks Road, Oxford, \\ OX1 3PJ, UK \\ ${ }^{2}$ UCL Department Of Medical Physics and Bioengineering, Malet Place Engineering \\ Building, Gower Street, London, WC1E 6BT, UK \\ ${ }^{3}$ Computing Laboratory, University of Oxford, Parks Road, Oxford, OX1 3QD, UK \\ ${ }^{4}$ Department of Neuroanaesthesia and Neurocritical Care, The National Hospital for \\ Neurology and Neurosurgery, Queen Square, London, WC1N 3BG \\ E-mail: abr@robots.ox.ac.uk
}

\begin{abstract}
Wavelet cross-correlation (WCC) is used to analyze the relationship between low frequency oscillations in near infrared spectroscopy (NIRS) measured cerebral oxyhaemoglobin $\left(\mathrm{O}_{2} \mathrm{Hb}\right)$ and mean arterial blood pressure (MAP) in patients suffering from autonomic failure and age matched controls. Statistically significant differences are found in the wavelet scale of maximum cross correlation upon posture change in patients, but not in controls. We propose that WCC analysis of the relationship between $\mathrm{O}_{2} \mathrm{Hb}$ and MAP provides a useful method of investigating the dynamics of cerebral autoregulation using the spontaneous low frequency oscillations that are typically observed in both variables without having to make the assumption of stationarity of the time series. It is suggested that for a short duration clinical test previous transfer function based approaches to analyze this relationship may suffer due to the inherent nonstationarity of low frequency oscillations that are observed in the resting brain.
\end{abstract}

Keywords: near infrared spectroscopy, time frequency analysis, wavelet cross correlation, posture changes, spontaneous oscillations, sympathetic stimulation, cerebral circulation. Submitted to: Physiol. Meas. 


\section{Introduction}

Cerebral autoregulation describes the regulatory response of cerebral haemodynamic variables to changes in blood pressure and is thought to be impaired in patients suffering from failure of the sympathetic nervous system (Panerai, 1998). An analysis technique is desired which can quantify the status of cerebral autoregulation from the often noisy and short duration measures of cardiovascular and haemodynamic variables obtained in the course of a head up tilt table test, a routine clinical procedure for the assessment of patients suffering from sympathetic impairment (Jaradeh and Prieto, 2003). Previous attempts have been made to quantify cerebral autoregulation in these patients by looking at absolute changes in cerebral blood flow and mean arterial pressure (for a comprehensive review see Panerai, 1998); however the noise that is inherent in the data means that repetitions of the test would be required for a single patient study. This is neither practical nor ethical in the case of patients suffering from failure of the autonomic nervous system (Panerai, 1998). This has led to a body of literature attempting to assess the status of autoregulation by considering the dynamic relationship between the spontaneous oscillations in cerebral blood flow that are know to occur in the resting brain and their counterparts in mean arterial blood pressure and heart rate.

There are a wide range of mechanisms which are thought to lead to low frequency spontaneous oscillations in cerebral haemodynamics. The sources of these oscillations are poorly understood, and explanations for the source of an oscillation at a given frequency are multiple, and often controversial. Giller et al. (1999) reported very low frequency oscillations in cerebral blood flow (CBF) detected using transcranial ultrasonography (TCD) in humans, and identify frequency bands centered around $0.0064 \mathrm{~Hz}, 0.02 \mathrm{~Hz}$ and $0.037 \mathrm{~Hz}$ that are synchronous throughout the cerebral vascular bed, yet not related to oscillations in blood pressure or $\mathrm{CO}_{2}$. Hudetz et al. (1992) report oscillations in $\mathrm{CBF}$ measured using laser doppler flowmetry (LDF) within a frequency range of $0.06-0.1833 \mathrm{~Hz}$ in anesthetized rats when blood pressure is held constant below $90 \mathrm{mmHg}$. Mayhew et al. (1996) use reflected light imaging to investigate $0.1 \mathrm{~Hz}$ oscillations in the exposed cerebral cortex of rat and cat. Obrig et al. (2000) demonstrate that similar oscillations may be captured noninvasively in the human brain using near infrared spectroscopy (NIRS) to measure cerebral oxyhaemoglobin concentration $\left(\mathrm{O}_{2} \mathrm{Hb}\right)$ and Elwell et al. (1999) underlined the implications of these oscillations for functional activation studies. It is suggested that oscillations in this region can be attributed to spontaneous vasomotion of cerebral vessels which may become entrained throughout the vasculature (Haddock and Hill, 2005; Nilsson and Aalkjaer, 2003), and should not be confused with similar low frequency pressure waves (Nilsson and Aalkjaer, 2003).

Within the same frequency region, however, high variability in blood pressure and heart rate is also found. Low frequency cardiovascular variability in humans is typically observed within two frequency regions (Cohen and Taylor, 2002). The first is thought 
to be respiratory related and occurs at a frequency of around $0.3 \mathrm{~Hz}$. The second is thought to be due to the action of the sympathetic nervous system and is centred at around 0.1Hz (Stauss et al., 1998; Malliani et al., 1991; Pagani et al., 1986; Nilsson and Aalkjaer, 2003; Cohen and Taylor, 2002). Mayer (1876) was the first to report slow waves in blood pressure at around this frequency hence cardiovascular oscillations in this frequency region are often termed Mayer, or M-Waves. Impairment of the action of the sympathetic nervous system has been shown in a number of studies to reduce the M-Wave component of cardiovascular variability (Elghozi et al., 1991; Chandler and Mathias, 2002; Cencetti et al., 1999; Kamiya et al., 2005; Kawaguchi et al., 2001). Since impairment of the sympathetic nervous system is observed in elderly patients, and associated with unexplained falls and syncope, bivariate relationships have been investigated between these oscillations in blood pressure and cerebral haemodynamics typically using transfer function identification techniques (Panerai et al., 1998; Simpson et al., 2001; Panerai et al., 2001; Mitsis and Marmarelis, 2002; Mitsis et al., 2002, 2004), and comparison of power spectral densities Tachtsidis et al. (2003, 2004). Tachtsidis et al. (2003) report changes in low frequency power of the spontaneous oscillations measured using NIRS with posture suggesting sympathetic influence on this variability.

The relationship between spontaneous low frequency oscillations in the resting brain and cardiovascular variability currently appears to be the most promising technique of assessing the status of cerebral autoregulation noninvasively in patients, yet there are numerous sources which may give rise to this variability, not all of which can be attributed to variations in cardiovascular parameters. It has been suggested by a number of other researchers (Giller and Mueller, 2003; Latka et al., 2005; Hu et al., 2006) that this could lead to a highly nonstationary relationship between cardiovascular and cerebral variability. Spontaneous oscillations have been assessed using a variety of modalities. The two prevalent non-invasive techniques for human studies are TCD and NIRS. TCD measures the velocity (VMCA) of blood flowing in the middle cerebral artery, whereas NIRS measures (among other things) an averaged tissue concentration $\left(\mathrm{O}_{2} \mathrm{Hb}\right)$ in the illuminated region which consists of arterial, arteriolar, capillary and venous blood flow.

In this paper, the complex continuous Morlet wavelet transform is used to perform a multiresolution analysis of mean arterial blood pressure and NIRS measured $\mathrm{O}_{2} \mathrm{Hb}$ time series taken from sympathetic failure patients and age matched controls undergoing passive posture changes induced by a head up tilt table test. Cross correlation is then used to derive a measure of linear relatedness between these variables as a function of wavelet scale (analogous to frequency) and time. This technique has been used for the assessment of coupling and synchronization between nonstationary geophysical oscillations (Grinsted et al., 2004) and in the EEG (Mizuno-Matsumoto et al., 2005) yet not for the assessment of autoregulation using NIRS. Since this technique does not make the assumption of stationarity for the relationship between the two time series it is suggested that it may provide a method which is more effective than the transfer function estimation techniques typically used for the assessment of cerebral 
autoregulation, particularly when used with time series obtained from short-duration head up tilt table tests.

\section{Clinical Methods}

The study utilised anonymised data from a previous investigation by our group in which changes in cerebral haemodynamics were measured with near infrared spectroscopy during orthostatic hypotension (Tachtsidis et al., 2005). The original study, approved by the Joint Research Ethics Committee of the National Hospital for Neurology and Neurosurgery and Institute of Neurology, included 8 patients with autonomic failure (Schatz et al., 1996) of mean age 42-79 years and 8 healthy age matched controls. Written consent was obtained from all participants prior to inclusion in the study. Passive head up tilt using a tilt table is a routine diagnostic test in patients with autonomic failure (Jaradeh and Prieto, 2003) and the study protocol in patients and controls consisted of 10 minutes of supine rest followed by 10 minutes of passive head up tilt and a further 10 minutes of supine rest. The head up tilt was reversed before 10 minutes if a patient developed syncope or pre-syncopal symptoms.

The continuous blood pressure waveform was monitored non-invasively for the duration

of the test using finger photoplethysmography (Portapres ${ }^{\circledR}$, Ohmeda) at a sampling frequency of $100 \mathrm{~Hz}$. A single channel continuous wave near-infrafred spectrometer with a sampling rate of $6 \mathrm{~Hz}$ (NIRO 300, Hamamatsu Photonics KK) was used to measure changes in $\mathrm{O}_{2} \mathrm{Hb}$ using the modified Beer-Lambert law (Delpy and Cope, 1997).

\section{Data Analysis}

The measured blood pressure (BP) time series was converted to MAP using an automated peak and trough detection algorithm (Townsend and Germuska, 2005), involving an adaptive rule-based search for the large gradient in blood pressure due to systole followed by waveform integration over the cardiac cycle. MAP and $\mathrm{O}_{2} \mathrm{Hb}$ were resampled to $10 \mathrm{~Hz}$ using cubic spline interpolation. Sections of the time series having a duration of 400 seconds were selected manually for each posture to avoid transient effects caused by the subject or clinicians adjusting the subject's posture during the tilt phase. Each section of data was then high pass filtered using a 5th order Butterworth filter with a cut-off frequency of $0.005 \mathrm{~Hz}$ to remove very slow variations and baseline shift (Mitsis et al., 2004) and subsequently low pass filtered using a 5th order Butterworth filter with a cut-off frequency of $0.8 \mathrm{~Hz}$ in order to remove variability in the signal due to the cardiac cycle. This preprocessing algorithm resulted in 3 pairs of preprocessed time series of 400 seconds duration at $10 \mathrm{~Hz}$ for each patient (MAP and $\mathrm{O}_{2} \mathrm{Hb}$ before, during and after the tilt). 
Figure 1 Time series measured from patients before and after pre-processing.

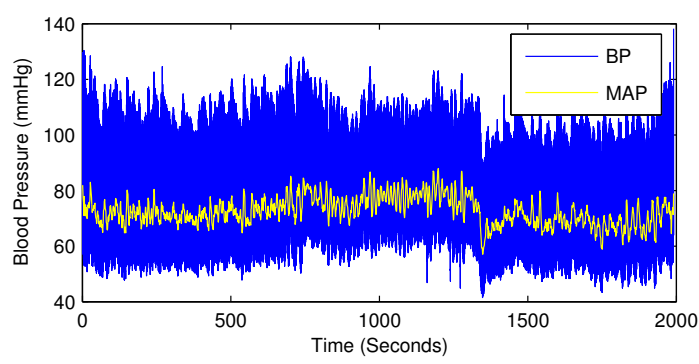

(a) Measured blood pressure (BP) and mean arterial pressure (MAP) time series.

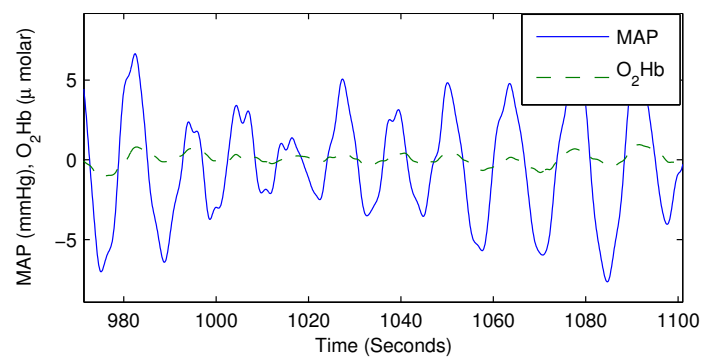

(c) Comparison of MAP and $\mathrm{O}_{2} \mathrm{Hb}$ time series indicating observed LF synchronization.

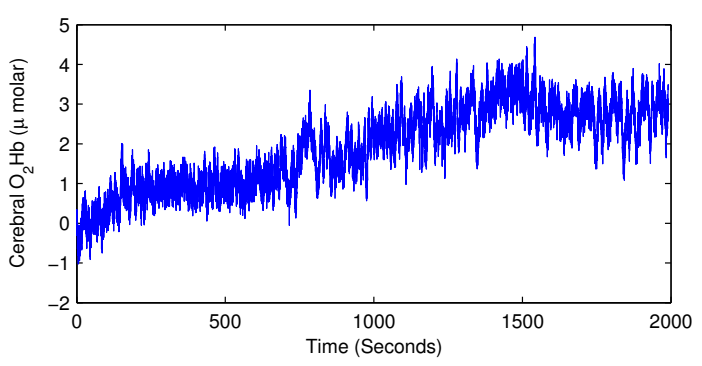

(b) Measured $\mathrm{O}_{2} \mathrm{Hb}$ time series.

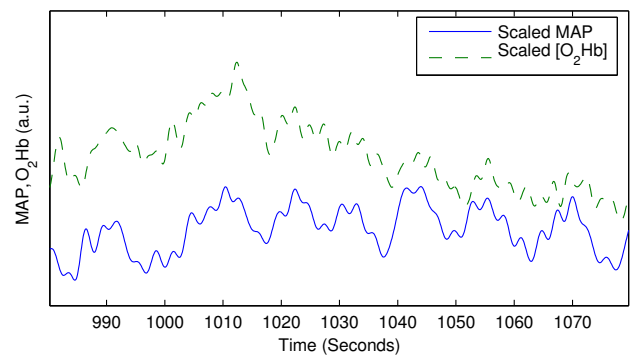

(d) Comparison of MAP and $\mathrm{O}_{2} \mathrm{Hb}$ time series indicating observed VLF synchronization.

Typical time series before and after pre-processing are shown in Figure 1. Figures 1(a) and 1(b) show typical records for the entire test duration. Figures 1(c) and 1(d) show short sections of the pre-processed time series that have been extracted to indicate the variability that is typically encountered. Figure 1(c) shows low frequency (LF) oscillations having a period close to 10 seconds in both the MAP and $\mathrm{O}_{2} \mathrm{Hb}$ time series. A similar LF variation can be seen in Figure 1(d) but with an additional very-lowfrequency (VLF) variation. It should be noted that in Figure $1(\mathrm{~d})$ the measured $\mathrm{O}_{2} \mathrm{Hb}$ has been scaled by a factor of 10 and offset vertically to illustrate the synchronization between the VLF component of the oscillation in the two time series.

Power spectral densities were calculated for each of the preprocessed time series with the Welch technique (Welch, 1967) with a 512-point (51.2 seconds) Hanning window with an overlap of 256 -points. Total power in the low-frequency $(0.005$ to $0.15 \mathrm{~Hz})$ and high frequency $(0.15$ to $0.5 \mathrm{~Hz})$ regions of the spectrum were obtained by trapezoidal integration of the power spectral density.

Continuous complex Morlet wavelet transforms with centre frequency $f_{c}=1$ were then computed for each time series at scales, $a$, with unit spacing from 20 to 250 (representing frequencies $0.5 \mathrm{~Hz}$ to $0.04 \mathrm{~Hz}$ for time series sampled at $10 \mathrm{~Hz}$ ) using the MATLAB ${ }^{\circledR}$ (Mathworks, Natick, MA.) wavelet toolbox function cwt (Misiti et al., 2004). This gave two complex time series, $W_{M A P}(a, t)$ and $W_{O H b}(a, t)$, for each segment of the test. Taking the complex argument of these time series gives a representation of the 
instantaneous phases, $\phi_{M A P}(a, t)$ and $\phi_{O H b}(a, t)$, as a function of wavelet scale and time (Le Van Quyen et al., 2001). Taking the difference allows the instantaneous phase difference to be calculated as:

$$
\Delta \phi(a, t)=\phi_{M A P}(a, t)-\phi_{O H b}(a, t) .
$$

To analyze synchronization between the MAP and $\mathrm{O}_{2} \mathrm{Hb}$ time series, the circular mean, $\overline{\Delta \phi(a)}$ of the phase difference over the duration of a test segment was calculated using:

$$
\overline{\Delta \phi(a)}=\tan ^{-1}\left(\frac{\sum_{t} \sin (\Delta \phi(a, t))}{\sum_{t} \cos (\Delta \phi(a, t))}\right) .
$$

The synchronization index, $\gamma(a)$ (Latka et al., 2005), which is an inverse circular statistical analogue of variance (see Fisher, 1995) was calculated using:

$$
\gamma(a)=\frac{1}{N}\left(\left[\sum_{t} \cos (\Delta \phi(a, t))\right]^{2}+\left[\sum_{t} \sin (\Delta \phi(a, t))\right]^{2}\right),
$$

in which $N$ is the number of time points in the series. The normalized wavelet crosscorrelation $\overline{W C C}(a, \tau)$ was calculated using:

$$
\overline{W C C}(a, \tau)=\frac{\left|R_{X, Y}\left(W_{M A P}, W_{O H b}, a, \tau\right)\right|}{\sqrt{\left|R_{X, X}\left(W_{M A P}, a, 0\right) \cdot R_{X, X}\left(W_{O H b}, a, 0\right)\right|}},
$$

in which $R_{X, Y}(s 1, s 2, a, \tau)$ denotes the cross-correlation of the wavelet coefficients of time series $s 1$ and $s 2$ at a scale $a$ for a relative time shift $\tau$ and $R_{X, X}(s 1, a, \tau)$ denotes the auto-correlation of the time series $s 1$ for a time shift $\tau$.

$\overline{W C C}(a, \tau)$ represents the cross-spectral power in the two time series (shifted relative to each other by $\tau$ ) as a fraction of the total power in the two time series. At a given wavelet scale $\overline{W C C}(a, \tau)=1$ would indicate that the coefficients of the two

wavelet transforms were related to each-other by a simple scaling factor, suggesting strong synchronization at this frequency. For a more detailed explanation of wavelet cross-correlation see Grinsted et al. (2004) and Maraun and Kurths (2004).

For each time series pair at each posture, the maximum value of $\overline{W C C}(a, \tau)$ was found, and the scale, $a_{M A X}$ and time delay, $\tau_{M A X}$ at which this maximum occurred was recorded. For this scale of maximum wavelet cross-correlation, the values of the circular mean phase difference, $\overline{\Delta \phi\left(a_{M A X}\right)}$, and the synchronization index, $\gamma\left(a_{M A X}\right)$, were also calculated. A paired t-test (see Goulden, 1959) was then used to analyze the statistical significance of the differences in the group mean of each of these variables between controls and patients, and with posture changes.

\section{Results}

Figure 2 shows filled contour plots of the wavelet cross-correlation function $\overline{W C C}(a, \tau)$ for a typical control subject and an autonomic failure patient during supine rest and 
Figure 2 Wavelet Cross Correlation between MAP and $\mathrm{O}_{2} \mathrm{Hb}$ for a typical control subject and an autonomic failure patient

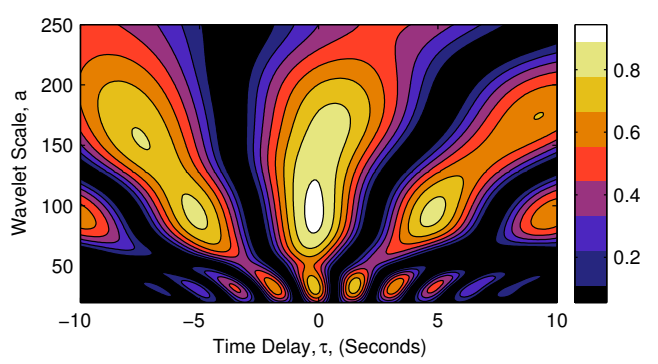

(a) Healthy Subject, Supine

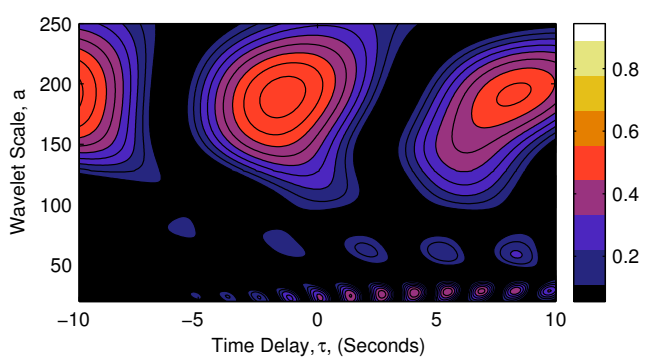

(c) Autonomic Failure Patient, Supine

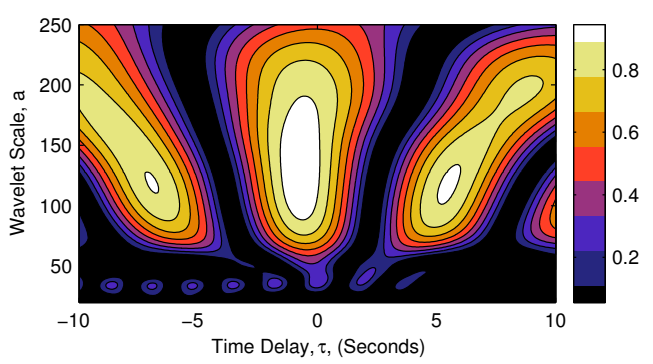

(b) Healthy Subject, Headup Tilt

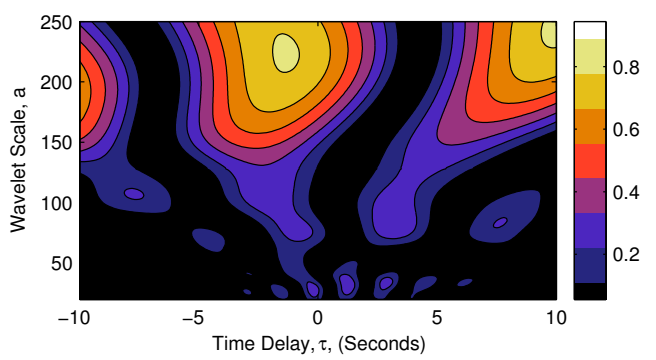

(d) Autonomic Failure Patient, Headup Tilt

head-up tilt.

For healthy subjects in the supine position before head-up tilt (Figure 2(a)) peaks in the wavelet cross-correlation typically occur within two regions. The first peak typically occurs at a scale of around $30(0.33 \mathrm{~Hz})$ and possibly corresponds to the respiratory peak. The second peak typically occurs at a scale of around $100(0.1 \mathrm{~Hz})$ which could correspond to the Mayer Wave peak. These results are in agreement with those of Latka et al. (2005). Upon head-up tilt in healthy subjects (Figure 2(b)) the level of cross correlation at the respiratory peak reduces significantly, and the Mayer Wave peak appears to spread to higher scales. There appears to be no discernible differences in the value of lag times at which these peaks occur.

In autonomic failure patients in the supine position (Figure 2(c)) no respiratory peak is typically observed in the wavelet cross-correlation, and the most significant peak appears to be at a much higher scale of around $180(\simeq 0.05 \mathrm{~Hz})$. The difference between the supine and head up wavelet cross correlation (Figures 2(c) and 2(d)) appears more subtle for this patient. This point will be revisited in discussion of the group statistics.

Figure 3 shows power spectral density plots for the same subjects as displayed in Figure 2. Figure 3(a) shows that power in the pre-tilt MAP time series for the healthy subject exists in the low frequency region, for frequencies less than around $0.15 \mathrm{~Hz}$, power in $\mathrm{O}_{2} \mathrm{Hb}$ for this subject (Figure 3(b)) shows a similar distribution however with a peak around $0.05 \mathrm{~Hz}$ that is slightly more pronounced than for MAP. For the head-up time 
Figure 3 Power Spectral Density of MAP and $\mathrm{OHb}$ for a typical control subject and an autonomic failure patient.

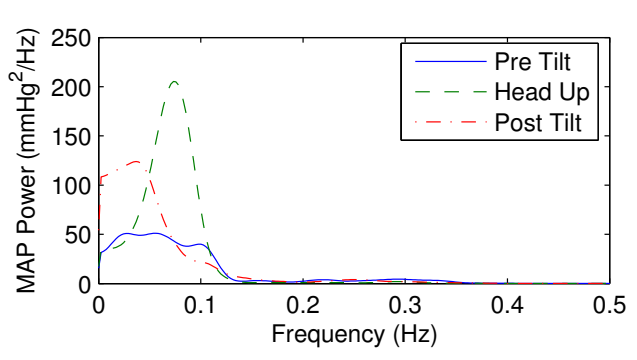

(a) Healthy Subject MAP

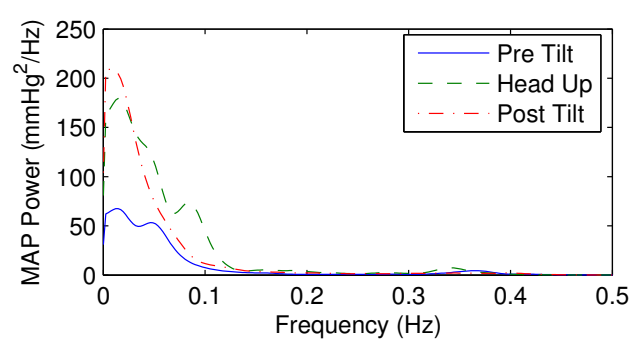

(c) Autonomic Failure Patient MAP

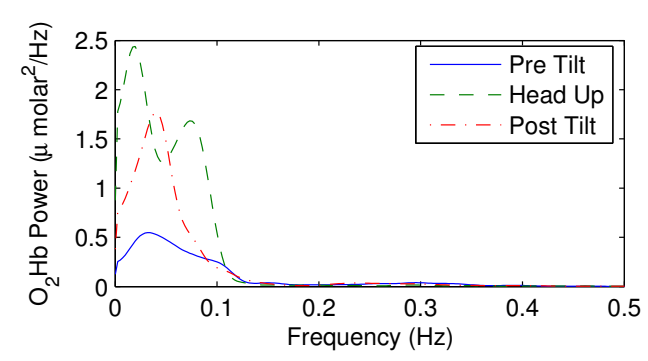

(b) Healthy Subject $\mathrm{O}_{2} \mathrm{Hb}$

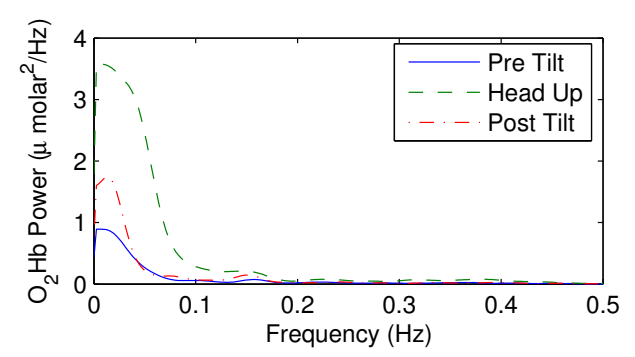

(d) Autonomic Failure Patient $\mathrm{O}_{2} \mathrm{Hb}$

series in the healthy subject spectral power in MAP (Figure 3(a)) shows a strong peak close to $0.1 \mathrm{~Hz}$, which is mirrored in the corresponding $\mathrm{O}_{2} \mathrm{Hb}$ spectrum (Figure $3(\mathrm{~b})$ ). The latter also shows an additional low frequency peak that has shifted to a lower frequency than in the pre-tilt $\mathrm{O}_{2} \mathrm{Hb}$ spectrum. The post-tilt time series in both MAP and $\mathrm{O}_{2} \mathrm{Hb}$ for the healthy subject show a single peak in the power spectrum which is at a similar frequency to the peak in the pre tilt case, albeit at higher power. It should be noted that peaks at frequencies higher than around $0.15 \mathrm{~Hz}$ are not significant in any of the power spectra.

For the autonomic failure patient (Figures $3(\mathrm{c})$ and $3(\mathrm{~d})$ ) peaks in the power spectra tend to be most pronounced at lower frequencies $(<0.05 \mathrm{~Hz})$ than in the healthy subject. For the the MAP time series (Figure 3(c)), both the pre-tilt and head-up power spectra show a secondary peak at slightly higher frequencies, however this is not reflected in either of the $\mathrm{O}_{2} \mathrm{Hb}$ spectra.

Figure 4 shows the value of the wavelet cross-correlation between $\mathrm{MAP}$ and $\mathrm{O}_{2} \mathrm{Hb}$ at a lag time of zero plotted versus frequency for direct comparison with the power spectral density plots in Figure 3. For the control subject (Figure 4(a)) we see two peaks, the first corresponding to a frequency of $0.1 \mathrm{~Hz}$, the second to a frequency of $\sim 0.3 \mathrm{~Hz}$. These results for healthy elderly control subjects are in general agreement with the results of Latka et al. (2005) for younger control subjects. Head-up tilt in the control subject causes the value of wavelet cross-correlation at the $0.3 \mathrm{~Hz}$ peak to diminish. For the autonomic failure patient (Figure $4(\mathrm{~b})$ ) the strongest peak in wavelet cross-correlation 
Figure 4 Value of wavelet cross-correlation between MAP and $\mathrm{O}_{2} \mathrm{Hb}$ at zero time delay for a typical control subject and an autonomic failure patient. For comparison with Figure 3, the wavelet scale has been converted to frequency using $f=\frac{10}{a}$.

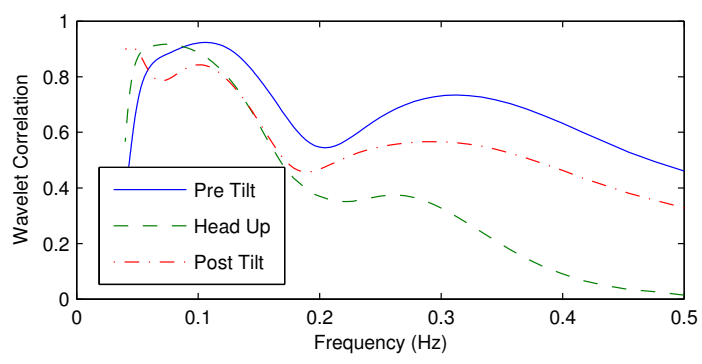

(a) Healthy Subject

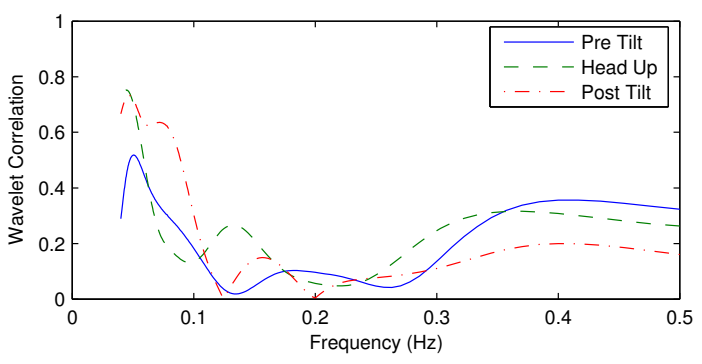

(b) Autonomic Failure Patient

appears at a frequency of around $0.05 \mathrm{~Hz}$, for all postures. Head-up tilt causes the value of the wavelet cross-correlation at this frequency to increase. An important difference between the wavelet synchronization analysis and power spectral density analysis can be illustrated by considering the peak that is typically observed at the respiratory frequency of $0.3 \mathrm{~Hz}$. Figure 3 shows no discernible peaks in the power spectral density of $\mathrm{O}_{2} \mathrm{Hb}$ or MAP at $0.3 \mathrm{~Hz}$, whereas Figure 4 shows strong values of wavelet cross-correlation in this frequency range. Wavelet cross-correlation is a measure of the level of similarity between two signals in a given frequency band and does not depend upon the power of those signals provided that the time series are compared for a sufficiently long time interval.

Figure 5 shows the mean and standard deviation for indices obtained from the wavelet cross-correlation analysis for both subject groups at each posture. Figure 5(a) shows that for the control subjects a statistically significant increase in the mean value of scale at maximum wavelet cross-correlation occurs on assuming the upright posture $(p=0.0038)$. Upon returning to the supine position after the tilt the group mean of the scales of maximum wavelet cross-correlation decreases again to a level similar to the pre-tilt value. In patients suffering from autonomic failure this change is not observed $(p=0.9895)$.

Figure 5(b) shows the value of the maximum wavelet cross-correlation. In all test subjects this was observed to be greater than 0.5. It can be seen that the value is typically lower in autonomic failure patients than in healthy subjects $(p=0.0238$ patient pre-tilt vs. control pre-tilt) although it increases on head-up tilt for patients $(p=0.0236)$, returning to a level similar to the pre-tilt value after the tilt. In healthy subjects this change is not observed.

Figure 5(c) shows the synchronization index (Equation 3) statistics for all subjects in the study. No significant differences between subject groups or with posture can be observed, although it should be noted that this index is again typically above 0.5 
Figure 5 Group statistical analysis at maximum wavelet correlation of scale, value of correlation, synchronization index and circular mean phase difference. Error bars indicate standard deviation.

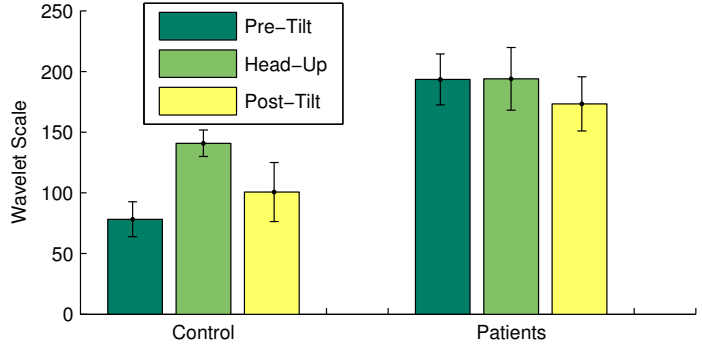

(a) Wavelet scale

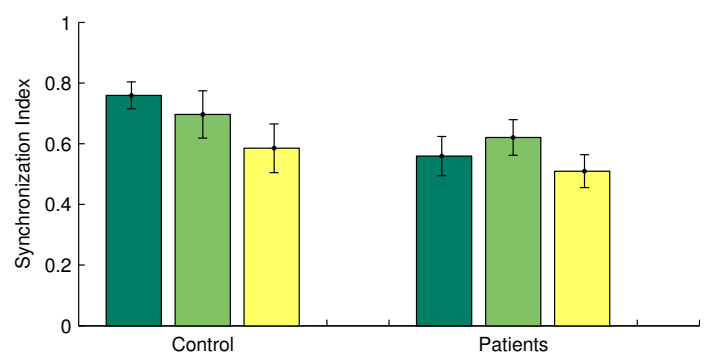

(c) Synchronization index

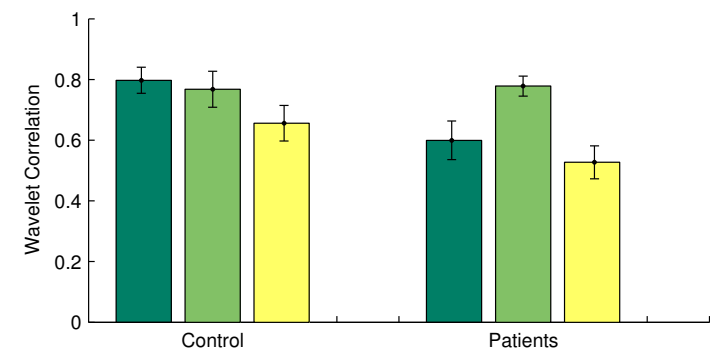

(b) Maximum wavelet correlation

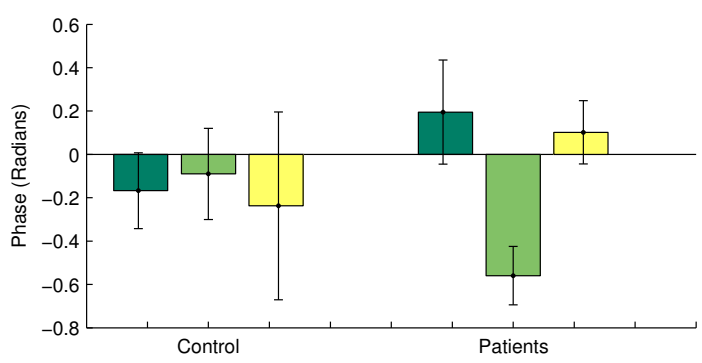

(d) Circular mean phase

indicating synchronization at this scale.

Figure 5(d) shows the group statistics for the circular mean phase difference between MAP and $\mathrm{O}_{2} \mathrm{Hb}$ (Equation 2). The variance in this index is quite large, illustrating its variability across subjects and with posture. Interestingly for control subjects the group mean is negative for all postures, whereas for sympathetic failure patients the mean is negative only during head-up tilt ( $p=0.0145$ patient pre-tilt vs. patient head-up; $p=0.0043$ patient head-up vs. patient post-tilt). A negative mean phase indicates that oscillations in $\mathrm{O}_{2} \mathrm{Hb}$ occur before those in MAP. This finding, although controversial, is in agreement with the results of Cencetti et al. (1999) and Zhang et al. (2002).

Figure 6 shows the group statistics for the power spectral density analysis. The large error bars indicate the variability that is typically encountered in power spectra measured from patients and controls. The only statistically significant differences were the increase in $\mathrm{LF} \mathrm{O}_{2} \mathrm{Hb}$ power for autonomic failure patients $(p=0.0011)$ and controls $(p=0.0234)$ when assuming the head-up posture. This was previously reported in a different study (Tachtsidis et al., 2004). It is possible that confidence in the results obtained from power spectral analysis could be increased with a longer duration test and a larger patient sample. 
Figure 6 Group statistical analysis of low frequency $(<0.15 \mathrm{~Hz})$, and high frequency $(>0.15 \mathrm{~Hz})$ spectral power in MAP and $\mathrm{O}_{2} \mathrm{Hb}$. Error bars indicate standard deviation.

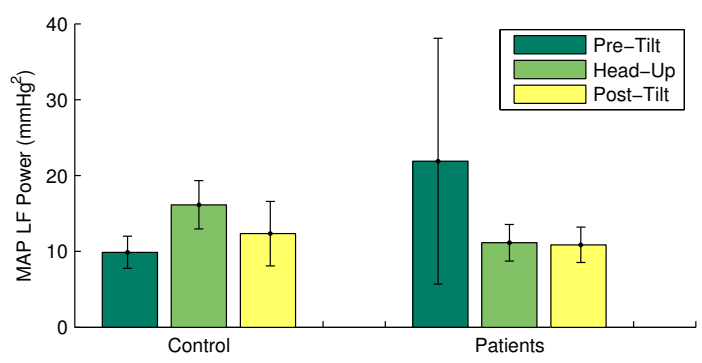

(a) LF MAP Total Power

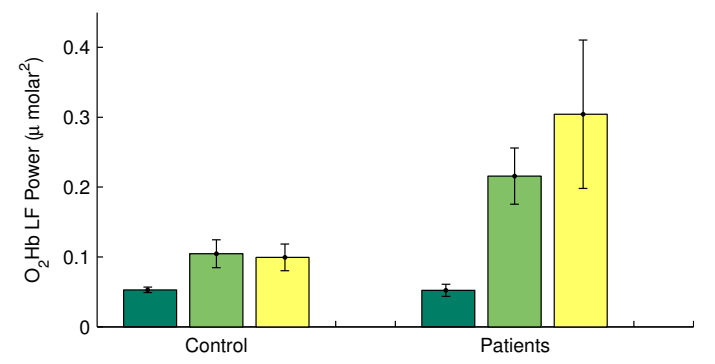

(c) $\mathrm{LF}_{2} \mathrm{Hb}$ Total Power

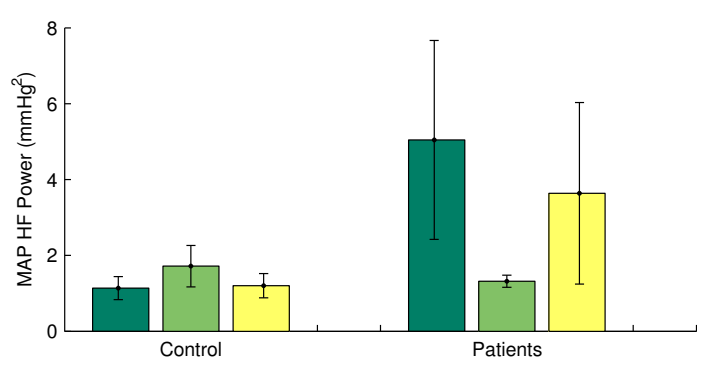

(b) HF MAP Total Power

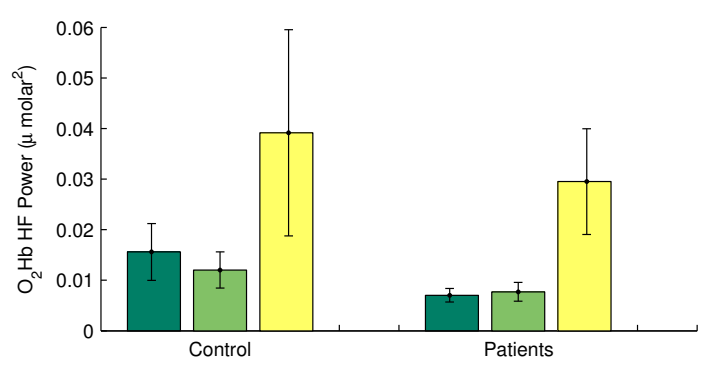

(d) $\mathrm{HF} \mathrm{O}_{2} \mathrm{Hb}$ Total Power

\section{Discussion}

Wavelet cross correlation (WCC) between MAP and $\mathrm{O}_{2} \mathrm{Hb}$ time series has been computed for elderly autonomic failure patients and age matched controls in the supine position and during head up tilt using a tilt table test. For healthy subjects in the supine position WCC typically exhibits two peaks, the first at a scale corresponding to a frequency of around $0.33 \mathrm{~Hz}$, the second at a frequency of around $0.1 \mathrm{~Hz}$. In healthy subjects passive head-up tilt using a tilt table causes the $0.33 \mathrm{~Hz}$ peak to disappear and the $0.1 \mathrm{~Hz}$ peak to shift to higher wavelet scales $(p=0.0038)$. For autonomic failure patients in the supine position the $0.33 \mathrm{~Hz}$ peak is not significant, and the LF peak typically occurs at a frequency lower than $0.1 \mathrm{~Hz}$. In patients, head-up tilt does not cause a statistically significant shift in the low frequency peak $(p=0.9859)$.

Power spectral analysis showed that increases in low frequency power in $\mathrm{O}_{2} \mathrm{Hb}$ can be observed in both patients $(p=0.0011)$ and controls $(p=0.0234)$ when they assume the upright posture in agreement with Tachtsidis et al. (2004). No statistically significant changes in LF MAP power were observed, probably reflecting the large variability observed in our patient group for LF MAP power (see the error bars in Figure 6(a)). It is not unreasonable to expect that this sort of variability is inherent to this patient group, for the short duration tests that are practical to obtain in a clinical setting.

Cerebral autoregulation broadly describes the mechanism which couples systemic 
variables (heart-rate, MAP) to cerebral haemodynamic variables (e.g. $\mathrm{O}_{2} \mathrm{Hb}$ ). This coupling is due to the cooperative action of both sympathetically mediated and local myogenic mechanisms. It is thought that myogenic mechanisms act to buffer small changes in cerebral blood flow due to changes in systemic variables, and that the sympathetic nervous system is most active during large pressure changes (Harper et al., 1972). In autonomic failure patients it is possible that the sympathetic mechanisms of cerebral autoregulation are impaired, and investigation of the relationship between systemic variables and cerebral haemodynamics could provide valuable diagnostic information in these patients. The inherent problem is that both systems exhibit considerable low frequency variability and it is difficult to attribute the frequency content of the signals to any one mechanism even within a narrow frequency band. This may be illustrated by considering Figures 3(a) and 3(c): in the low frequency region MAP shows relatively broad spectral content below $0.1 \mathrm{~Hz}$ in both controls and autonomic failure patients. Figures $3(\mathrm{~b})$ and $3(\mathrm{~d})$ show that the $\mathrm{O}_{2} \mathrm{Hb}$ frequency content is similarly variable in this range.

Many previous attempts to quantify the dynamics of cerebral autoregulation (see Panerai, 1998) have done so using a system identification technique which essentially involves division of the power spectra of MAP and TCD-measured VMCA resulting in a complex transfer function expressing the gain and phase of the system as a function of frequency. This makes the assumption that the entire frequency content of cerebral haemodynamic variables can be attributed to driving by systemic frequency content. The assumption of stationarity (that the relationship between the two time series remains relatively constant over time) is also required. Recent studies demonstrating changes in TCD-measured VMCA during functional studies (Panerai et al., 2005) underline the limitations of these assumptions. It is also reasonable to expect that in autonomic failure patients in a clinical setting the assumption of stationarity could be confounded by the effect of experimental noise, and the need to take a very short duration test in order to minimize patient discomfort.

Wavelet cross-correlation decomposes the MAP and $\mathrm{O}_{2} \mathrm{Hb}$ signals into wavelet modes which are highly localized in frequency and allows investigation of which modes are most linearly related. It makes no assumption about the stationarity of the relationship between the time series, and if other modes in cerebral haemodynamics exist which are not the result of driving by systemic variables these will show a low value of wavelet cross-correlation. Investigation of the scale at which wavelet cross-correlation has its maximum has shown that on head-up tilt in healthy subjects the frequency at which maximum wavelet cross correlation occurs decreases. In autonomic failure patients this frequency is lower than in controls, and does not show a statistically significant shift with posture. Consideration of the circular mean phase measured using wavelet analysis has shown that in controls, $\mathrm{O}_{2} \mathrm{Hb}$ typically leads MAP, and shows no statistically significant differences with posture. In patients suffering from failure of the sympathetic nervous system in the supine position, $\mathrm{O}_{2} \mathrm{Hb}$ phase lags MAP phase however when the patients 
assume the upright posture, $\mathrm{O}_{2} \mathrm{Hb}$ shows a statistically significant phase lead with respect to MAP.

These results may be explained with reference to the physiology of the patient groups as follows; cardiovascular oscillations around a frequency of $0.1 \mathrm{~Hz}$ are thought to be a marker of sympathetic control of circulation. The observed phase lead of the wavelet components of $\mathrm{O}_{2} \mathrm{Hb}$ with respect to MAP at maximum cross-correlation in control subjects for all postures in the supine posture suggests that these components reflect neural control of the cerebral circulation. In patients in the supine position $\mathrm{O}_{2} \mathrm{Hb}$ lagging MAP indicates passive stimulation of the cerebral circulation by low frequency content in blood pressure (Cencetti et al., 1999). When patients assume the upright posture, this does not cause the frequency of maximum correlation to change much, however phase changes at this frequency from $\mathrm{O}_{2} \mathrm{Hb}$ lagging MAP to $\mathrm{O}_{2} \mathrm{Hb}$ leading MAP, as is the case for control subjects in all postures. In control subjects the statistically significant shift in the scale of maximum correlation indicates that the sympathetic mechanism is indeed challenged by the change in posture. However, the fact that the phase lead of $\mathrm{O}_{2} \mathrm{Hb}$ with respect to MAP is preserved throughout the test suggests that neural control is maintained even upon head-up tilt and explains why syncope is rarely observed in these patients. In sympathetic failure patients the $\mathrm{O}_{2} \mathrm{Hb}$ phase lag suggests impaired sympathetic regulation. On head up tilt some mechanism is activated to cause phase lead yet this is not sufficient in many cases to prevent syncope.

The results of this study for healthy subjects are in agreement with the results of Latka et al. (2005) who investigated synchronization between MAP and TCD-measured middle cerebral artery blood flow velocity using complex Morlet wavelets for 10 healthy young subjects and observed significantly reduced circular phase variance around $0.33 \mathrm{~Hz}$ and $0.1 \mathrm{~Hz}$. Our study represents the first time that wavelet cross-correlation has been used to investigate the posture dependence of synchronization between MAP and NIRSmeasured $\mathrm{O}_{2} \mathrm{Hb}$ in elderly autonomic failure patients in comparison with age-matched controls.

\section{Conclusion}

LF oscillations in cerebral $\mathrm{O}_{2} \mathrm{Hb}$ measured using single-channel NIRS have been related to LF oscillations in systemic MAP measured non-invasively using finger photoplethysmography by investigating wavelet cross-correlation between the two time series. This analysis has been performed for elderly patients suffering from autonomic failure and healthy age-matched controls. It has been shown that the frequency of maximum wavelet cross correlation is significantly different for patients with respect to controls. We argue that synchronization analysis provides information which is fundamentally different from the comparison of power spectra. 
Estimation of the phase relationship between the two sources of oscillation using the complex continuous wavelet transform has demonstrated the variability of this measure in the patient group considered. This suggests that the assumption of stationarity and transfer function analysis may produce confusing results in elderly subjects and those suffering from autonomic failure.

Investigation of the spectral properties of LF oscillations in cerebral haemodynamics and their relationship to other variables (both global cardiovascular variability and local cerebral effectors) is important in understanding the sources of physiological 'noise' in functional imaging experiments and in the diagnosis of patients with autonomic failure. It is suggested that further work should investigate the applicability of this technique to the analysis of low frequency synchronization of cerebral haemodynamics with other potential sources of low frequency variability in cardiovascular parameters, for example low frequency fluctuations in the partial pressure of end-tidal carbon dioxide $\left(\mathrm{PET}_{\mathrm{CO}_{2}}\right)$.

\section{Acknowledgments}

The authors would like to thank the staff of the Autonomic Unit at the National Hospital for Neurology and Neurosurgery and Dr Katharine Hunt for assistance in collection of the data and Professor Christopher Mathias for allowing us to include his patients in the original study.

The authors are pleased to acknowledge the support of the United Kingdom Research Councils through an EPSRC Life Sciences Interface Doctoral Training Centre studentship (GR/S58119/01) to A.B.R. and an EPSRC/MRC grant no GR/N14248/01 to I.T.

\section{References}

Cencetti S, Lagi A, Cipriani M, Fattorini L, Bandinelli G and Bernardi L 1999 Heart 82(3), 365-372.

Chandler M P and Mathias C J 2002 J Neurol 249(5), 542-548.

Cohen M A and Taylor J A 2002 J Physiol 542(Pt 3), 669-683.

Delpy D T and Cope M 1997 Philosophical Transactions of the Royal Society of London, B 352(1354), 649-659.

Elghozi J L, Laude D and Janvier F 1991 J Cardiovasc Pharmacol 17(6), 935-940.

Elwell C E, Springett R, Hillman E and Delpy D T 1999 Adv Exp Med Biol 471, 57-65.

Fisher N I 1995 Statistical Analysis of Circular Data Cambridge University Press. 
Giller C A, Hatab M R and Giller A M 1999 J Cereb Blood Flow Metab 19(4), 452-459.

Giller C A and Mueller M 2003 Med Eng Phys 25(8), 633-646.

Goulden C H 1959 Methods of statistical analysis (Wiley publications in statistics) Wiley.

Grinsted A, Moore J C and Jevrejeva S 2004 Nonlinear Processes in Geophysics 11, 561566.

Haddock R E and Hill C E 2005 The Journal of Physiology 566(3), 645-656.

Harper A M, Deshmukh V D, Rowan J O and Jennett W B 1972 Arch Neurol 27(1), 1-6.

Hu X, Nenov V, Glenn T C, Steiner L A, Czosnyka M, Bergsneider M and Martin N 2006 Biomedical Engineering, IEEE Transactions on 53(2), 195-209.

Hudetz A G, Roman R J and Harder D R 1992 J Cereb Blood Flow Metab 12(3), 491499.

Jaradeh S S and Prieto T E 2003 Phys Med Rehabil Clin N Am 14(2), 287-305.

Kamiya A, Hayano J, Kawada T, Michikami D, Yamamoto K, Ariumi H, Shimizu S, Uemura K, Miyamoto T, Aiba T, Sunagawa K and Sugimachi M 2005 Am J Physiol Heart Circ Physiol 289(4), H1758-H1769.

Kawaguchi T, Uyama O, Konishi M, Nishiyama T and Iida T $2001 \mathrm{~J}$ Gerontol A Biol Sci Med Sci 56(5), M273-M280.

Latka M, Turalska M, Glaubic-Latka M, Kolodziej W, Latka D and West B J J 2005 Am J Physiol Heart Circ Physiol 289, H2272-H2279.

Le Van Quyen M, Foucher J, Lachaux J, Rodriguez E, Lutz A, Martinerie J and Varela F J 2001 J Neurosci Methods 111(2), 83-98.

Malliani A, Pagani M, Lombardi F and Cerutti S 1991 Circulation 84(2), 482-492.

Maraun D and Kurths J 2004 Nonlin. Processes Geophys. 11, 505-514.

Mayer S 1876 Sitzungsberichte Akademie der Wissenschaften in Wien. Mathematischnaturwissenschaftliche Classe, Anatomie 74, 281-307.

Mayhew J E, Askew S, Zheng Y, Porrill J, Westby G W, Redgrave P, Rector D M and Harper R M 1996 Neuroimage 4(3 Pt 1), 183-193.

Misiti M, Misiti Y, Oppenheim G and Poggi J M 2004 Matlab Wavelet Toolbox User's Guide. Version 3. The Mathworks, Inc. Natick, MA.

Mitsis G D, Poulin M J, Robbins P A and Marmarelis V Z 2004 IEEE Trans Biomed Eng 51(11), 1932-1943.

Mitsis G and Marmarelis V 2002 Ann Biomed Eng 30(2), 272-81. 
Mitsis G, Zhang R, Levine B and Marmarelis V 2002 Ann Biomed Eng 30(4), 555-65.

Mizuno-Matsumoto Y, Ukai S, Ishii R, Date S, Kaishima T, Shinosaki K, Shimojo S, Takeda M, Tamura S and Inouye T 2005 Brain Topogr 17(4), 237-252.

Nilsson H and Aalkjaer C 2003 Mol Interv 3(2), 79-89.

Obrig H, Neufang M, Wenzel R, Kohl M, Steinbrink J, Einhupl K and Villringer A 2000 Neuroimage 12(6), 623-639.

Pagani M, Lombardi F, Guzzetti S, Rimoldi O, Furlan R, Pizzinelli P, Sandrone G, Malfatto G, Dell'Orto S and Piccaluga E 1986 Circ Res 59(2), 178-193.

Panerai R B 1998 Physiol Meas 19(3), 305-338.

Panerai R B, Dawson S L, Eames P J and Potter J F 2001 Am J Physiol Heart Circ Physiol 280(5), H2162-H2174.

Panerai R B, Moody M, Eames P J and Potter J F 2005 J Appl Physiol 99(6), 23522362.

Panerai R B, White R P, Markus H S and Evans D H 1998 Stroke 29(11), 2341-2346.

Schatz I J, Bannister R, Freeman R L, Goetz C G, Jankovic J, Kaufmann H C, Koller W C, Low P A, Mathias C J, Polinsky R J, Quinn N P, Roberston D and Streeten D H P 1996 J Neurol Sci 144(1-2), 218-219.

Simpson D M, Panerai R B, Evans D H and Naylor A R 2001 Ann Biomed Eng 29(1), 1825.

Stauss H M, Anderson E A, Haynes W G and Kregel K C 1998 Am J Physiol 274(4 Pt 2), H1277-H1283.

Tachtsidis I, Elwell C E, Lee C W, Leung T S, Smith M and Delpy D T 2003 Adv Exp Med Biol 540, 31-36.

Tachtsidis I, Elwell C E, Leung T S, Bleasdale-Barr K, Hunt K, Toms N, Smith M, Mathias C J and Delpy D T 2005 Adv Exp Med Biol 566, 187-193.

Tachtsidis I, Elwell C E, Leung T S, Lee C W, Smith M and Delpy D T 2004 Physiol Meas 25(2), 437-445.

Townsend N W and Germuska R B 2005 'Locating features in a photoplethysmoraph signal' US Patent Application 10/490,545.

Welch P D 1967 IEEE Transactions on Audio and Electroacoustics 15, 70-73.

Zhang R, Zuckerman J H, Iwasaki K, Wilson T E, Crandall C G and Levine B D 2002 Circulation 106(14), 1814-1820. 